Revue d'histoire de l'enfance " irrégulière »

Le Temps de l'histoire

15 | 2013

Enfances déplacées. (II) en temps de guerre

\title{
(Lecture de) Les lascars. Une jeunesse en colère
}

\section{Véronique Blanchard}

\section{(2) OpenEdition}

Journals

Édition électronique

URL : http://journals.openedition.org/rhei/3549

DOl : 10.4000/rhei.3549

ISSN : $1777-540 \mathrm{X}$

Éditeur

Presses universitaires de Rennes

Édition imprimée

Date de publication : 30 octobre 2013

Pagination : 218-221

ISBN : 978-2-7535-2896-3

ISSN : 1287-2431

Référence électronique

Véronique Blanchard, «(Lecture de) Les lascars. Une jeunesse en colère », Revue d'histoire de l'enfance " irrégulière » [En ligne], 15 | 2013, mis en ligne le 30 octobre 2013, consulté le 22 septembre 2020.

URL : http://journals.openedition.org/rhei/3549; DOI : https://doi.org/10.4000/rhei.3549

Ce document a été généré automatiquement le 22 septembre 2020.

(C) PUR 


\title{
(Lecture de) Les lascars. Une jeunesse en colère
}

\author{
Véronique Blanchard
}

\section{RÉFÉRENCE}

Éditions Autrement, collection « Frontières », 153 p. ISBN 9782746714786

1 Ce livre de moins de 200 pages est plus un traité philosophique et politique qu'un essai sociologique. Il offre une réflexion originale sur la jeunesse d'aujourd'hui et sur les actes transgressifs tant reprochés à ceux que Marc Hatzfeld surnomme les « lascars ».

2 Ce sociologue connaît bien son sujet, il est déjà l'auteur entre autres de La culture des cités : une énergie positive ${ }^{1}$. Ici, il effectue une enquête de terrain, de nombreux entretiens avec des jeunes rencontrés tant dans la banlieue francilienne, qu'en Bretagne et quelques bourgs ruraux. Il mêle son approche ethnographique à des références philosophiques (extraits de Spinoza ou Nietzsche), à des grands textes littéraires (À la recherche du temps perdu, Roméo et Juliette...), à quelques mythes grecs (Antigone, Prométhée...) et aussi à la filmographie des années 1950 (Les 400 coups, Los Olvidados...).

Dans les premiers chapitres, Marc Hazfeld n'échappe pas à l'exercice de définition de la jeunesse, mais il le fait en se centrant sur la jeunesse populaire, sur le rapport intime existant entre elle et la transgression, et enfin en s'interrogeant sur la place des adultes qui acceptent difficilement les tourbillons de la jeunesse.

4 Ses mots pour cerner les jeunes sont souvent plus poétiques, que sociologiques. Comme il le dit lui-même, il s'agit d'une "approche sensible ». Il montre comme c'est un âge interstitiel (p.34), un moment entre l'enfance et la maturité, qui se prolonge ces dernières décennies du fait de l'allongement de la vie et de la généralisation de la scolarité.

5 S'il se penche sur la jeunesse des milieux populaires, c'est parce que selon lui elle est plus tentée par la transgression du fait de sa proximité avec la violence, une violence réelle, très liée à un retour de la violence du travail prolétaire. Mais aussi parce que la 
précarité est une réalité indéniable des familles de ces jeunes. Marc Hazfeld insiste sur l'idée que "la délinquance de la jeunesse est immergée dans la nécessité » ${ }^{(\mathrm{p} .}{ }^{65)}$. Enfin l'auteur pointe le racisme latent dont ces adolescents sont victimes; ils sont perçus non seulement comme dangereux car appartenant aux classes laborieuses, mais aussi comme dangereux parce qu'ils ne sont pas considérés comme nos enfants, mais comme des étrangers ${ }^{2}$. Ces trois éléments expliquent que certains vivent «une forme de désespoir continu de faible intensité » (p. 66), les menant à la transgression.

Une des originalités de cet ouvrage porte sur la définition que donne l'auteur de la transgression, et en particulier de la transgression des adolescents. Premièrement, il insiste sur le fait qu'ils représentent la force vitale de notre société. Par essence ils sont, comme il le souligne, "turbulents ", «bouillonnants ", "tourbillonnants ». Ces excès, ce désordre, cette énergie les conduisent inévitablement à transgresser.

De plus, en faisant référence à des textes fondateurs (Adam et Ève, Prométhée, la tradition hindouiste), il démontre que la «transgression est le geste qui dévoile la conscience» (p.86), c'est-à-dire que la transgression possède un aspect positif, permettant la voie vers un ailleurs, offrant une autre vision du monde, voire même aidant à la mise en place de nouvelles règles. En reprenant des figures de la fiction comme le héros de l'Attrape-cœur, l'Antoine Doinel des 400 coups ou le James Dean de la Fureur de vivre, Marc Hatzfeld estime que les gestes transgressifs de la jeunesse devraient nous permettre d'apprendre à vivre. C'est pourquoi il explique qu'il est bien sûr nécessaire que la loi soit respectée mais aussi important de comprendre que parfois la jeunesse se doit de l'enfreindre.

Dans son quatrième chapitre, l'auteur prend nettement position, le ton est parfois même polémique concernant les fonctionnements de la justice. Il dénonce une loi qui ces dernières années a perdu, pour les classes populaires, son sens protecteur, qui souvent est perçue comme injuste et privilégiant, non plus l'intérêt collectif, mais l'intérêt particulier. Selon lui, cette loi est plus adaptée à ceux qui l'ont conçue, qu'à ceux qui la subissent. Tout un développement est consacré à une justice qui dorénavant console les victimes en escamotant la prévention, l'éducation et la punition (p.112). Marc Hatzfeld veut montrer que si les jeunes des milieux populaires sont considérés comme plus délinquants que les autres, c'est avant tout qu'ils subissent le «soupçon de transgression potentielle» (p.125), qui s'accompagne d'un fichage et d'une surveillance, entraînant mécaniquement plus d'arrestations. Il dénonce le tête-à-tête "pathétique entre les jeunes et la police» avec, dit-il, un «honneur à mater des enfants comme des tigres sauvages $"\left({ }^{p} .{ }^{147)}\right.$.

Enfin, l'auteur répète vigoureusement son idée que la jeunesse est par essence effrontée, que c'est un des éléments qui fait sa force et sa richesse. Cependant certains adultes perçoivent la transgression des jeunes, non pas comme une effronterie, mais comme un acte tragique, une atteinte à l'ordre. Il estime que ce malentendu initial, cette difficulté à la compréhension réciproque ne permet pas l'arrêt de la transgression. Et il conclut en précisant qu'il existe une multitude de désobéissances et qu'il y a donc une multitude de réponses sociales à la transgression, la punition ne devrait en être qu'une parmi d'autres. Il insiste, pour terminer, sur la nécessité de ne jamais oublier que le jeune transgresseur est un des nôtres, un représentant de la jeunesse, notre futur.

10 Ce livre revigorant par son ton et sa plume alerte, permet aux historiens de l'enfance irrégulière d'aborder le sujet de la délinquance juvénile sous un angle différent, 
puisque si l'approche n'est certes pas diachronique, elle est, grâce au regard de l'ethnologue, presque universelle.

\section{NOTES}

\section{Autrement, 2006}

2. Idée largement développée aussi dans le livre de Pierre JOXE, Pas de quartier? Délinquance juvénile et justice des mineurs, Fayard, 2012. 